\title{
ANÁLISE ESPECTRAL DE SÉRIES TEMPORAIS ATRAVÉS DE PONTOS FIXOS
}

\author{
Sílvia Lopes*
}

\section{Resumo}

Neste artigo consideramos modelos de espectro misto da forma

$$
Z_{t}=\sum_{j=1}^{p} A_{j} \cos \left(\omega_{j} t+\phi_{j}\right)+\xi_{t}, \quad t \in \mathbf{Z}
$$

onde $p$ não é necessariamente conhecido e, para cada $1 \leq j \leq p, A_{j}$ é constante desconhecida, $\omega_{j}$ é freqüência desconhecida em $(-\pi, \pi]$ e a fase $\phi_{j}$ é uma variável aleatória uniformemente distribuída em $(-\pi, \pi]$ independente cada uma entre si e do processo de ruído. O objetivo é estimar as freqüências da parte discreta do espectro. Esta estimativa é obtida através de um método recursivo cujos parâmetros são atualizados a cada passo. Os cosenos das freqüências são obtidos como pontos fixos atratores de uma determinada aplicação.

\section{Abstract}

This article analyzes the mixed spectrum stationary process

$$
Z_{t}=\sum_{j=1}^{p} A_{j} \cos \left(\omega_{j} t+\phi_{j}\right)+\xi_{t}, \quad t \in \mathbf{Z}
$$

where $p$ is not necessarily known, $A_{1}, \ldots, A_{p}$ are unknown constants, $\omega_{1}, \ldots, \omega_{p}$ are unknown frequencies with values in $(-\pi, \pi],\left\{\xi_{t}\right\}_{t \in \mathbf{Z}}$ is a white noise process with mean 0 and variance $\sigma_{\xi}^{2}$ and $\phi_{1}, \ldots, \phi_{p}$ are independent random variables uniformly distributed in $(-\pi, \pi]$ and are independent of the noise process. The goal of this paper is to estimate each discrete frequency. The estimate is obtained from a recursive method with updated parameters. The cosine of each discrete frequency is obtained as an attracting fixed point of a certain map.

\section{Introdução.}

Neste artigo vamos utilizar seqüências de filtros paramétricos com o objetivo de estimar as freqüências de um modelo de espectro

*Instituto de Matemática, Universidade Federal do Rio Grande do Sul.

R. de Econometria Rio de Janeiro v. 14, $\mathrm{n}^{2} 1$, p.43-70 abril 1994/outubro 1994 
misto. Isto é feito iterativamente, como segue. Uma série temporal é filtrada por um filtro paramétrico e a resultante função de autocorrelação de primeira ordem é imediatamente usada no ajuste do parâmetro do filtro. O filtro ajustado é então aplicado novamente, dando origem a uma nova autocorrelação de primeira ordem e o procedimento é repetido. Escolhendo filtros apropriados, o esquema produz seqüências convergentes e, sob certas condiçōes, este método garante a consistência forte dos estimadores do coseno das freqüências da parte discreta do espectro.

Para expressar as mesmas idéias através de uma linguagem matemática mais precisa, considere $\left\{Z_{t}\right\}_{t \in \mathbf{Z}}$ um processo estocástico estacionário de média zero e seja $\left\{\mathcal{L}_{\theta}(\cdot)\right\}_{\theta \in \Theta}$ uma família paramétrica de filtros lineares invariantes no tempo, onde $\theta$ é um parâmetro finitodimensional no espaço de parâmetros $\Theta$. Denotamos por $\left\{Z_{t}(\theta)\right\}_{t \in \mathbf{Z}}$ o processo filtrado

$$
Z_{t .}(\theta)=\mathcal{L}_{\theta}(Z)_{t} .
$$

Então $\{\rho(\theta)\}_{\theta \in \Theta}$, definida por

$$
\rho(\theta)=\frac{E\left[Z_{t}(\theta) Z_{t+1}(\theta)\right]}{E\left[Z_{t}^{2}(\theta)\right]}
$$

é uma família de autocorrelações de primeira ordem parametrizadas, quando $\left\{Z_{t}(\theta)\right\}_{t \in \mathbf{Z}}$ for um processo estocástico real.

Estamos interessados nos pontos fixos da aplicação $\rho(\theta)$ obtidos a partir da convergência da recursão

$$
\theta_{k+1}=\rho\left(\theta_{k}\right)=\rho^{k}\left(\theta_{0}\right)
$$

para algumas famílias específicas de filtros paramétricos.

O nosso objetivo é obter filtros paramétricos lineares de tal forma que os pontos fixos a serem determinados pela aplicação $\rho(\theta)$ resultante, coincidam com os cosenos das freqüências que desejamos estimar.

O método a ser apresentado nestas notas é um procedimento alternativo à Análise do Periodograma para a estimativa de freqüências em um modelo de espectro misto. Este método apresenta uma 
complexidade computacional da ordem de $O(N)$, onde $N$ é o tamanho amostral considerado (veja Yakowitz (1991)). Isto significa um avanço sobre o algorítmo da FFT que tem complexidade computacional da ordem de $O\left(N \log _{2} N\right)$. Este método que, em um certo sentido, lembra o Método de Newton para determinar as raízes de uma equação não-linear é chamado de Método da Contração na Deteç̧ão de Freqüencias (MC). Ele tem como uma de suas grandes vantagens, a não necessidade de informações a priori da localização aproximada das freqüências.

Os modelos de espectro misto, como foram mencionados acima, aparecem em várias áreas aplicadas como economia, oceanografia, engenharia, etc..

Estas notas foram escritas a partir de parte da Tese de Doutoramento da autora realizada na Universidade de Maryland, sob a orientação do Dr. Benjamin Kedem e dos artigos Kedem e Lopes (1992) e Lopes e Kedem (1994).

\section{O problema.}

Considere o modelo de espectro misto

$$
Z_{t}=\sum_{j=1}^{p} A_{j} \cos \left(\omega_{j} t+\phi_{j}\right)+\xi_{t}, \text { para } t \in \mathbf{Z}
$$

onde $p$ não é necessariamente conhecido e, para cada $j \in\{1,2, \ldots, p\}$, $A_{j}$ é uma constante desconhecida, $\omega_{j}$ é uma freqüência desconhecida tomando valor em $(-\pi, \pi]$ e a fase $\phi_{j}$ é uma variável aleatória uniformemente distribuída em $(-\pi, \pi]$ independente cada uma entre si e também da componente de ruído. Por simplicidade, assumimos aqui que esta componente é um ruído branco Gaussiano tal que $\xi_{t} \sim \mathcal{N}\left(0, \sigma_{\xi}^{2}\right)$. Esta hipótese de ruído branco não é necessária para o que segue mas ela simplifica a exposição. Na verdade, qualquer ruído que seja um processo estocástico estacionário e ergódico e com distribuição espectral contínua servirá bem.

Apresentamos aqui um método (ver Kedem e Lopes (1992) e Lopes e Kedem (1994)) inspirado no Algorítmo de He e Kedem (veja o trabalho de He e Kedem (1989)) que permite a obtenção, através de 
um procedimento iterativo e com alta ordem de precisão, dos valores estimados de $A_{j}, \omega_{j}, 1 \leq j \leq p$, e $\sigma_{\xi}$.

Em uma simulação com o modelo (2.1) quando $p=2, A_{1}=$ $A_{2}=1.0, \omega_{1}=0.7$ e $\omega_{2}=2.2$, e utilizando o método a ser explicado aqui, obtivemos os seguintes estimadores consistentes: $\hat{\omega}_{1}=0.7044$ e $\hat{\omega}_{2}=2.1965$. Neste artigo nos preocupamos apenas em explicar o método para a obtenção das freqüências $\omega_{j}$, para $1 \leq j \leq p$. Para a estimação dos demais parâmetros envolvidos no modelo (2.1), veja Lopes (1993).

Com a intenção de simplificar a exposição, nos concentramos primeiramente no Método da Contração na Deteç̧ão de Freqüências quando o modelo usado é do tipo (2.1) na situação mais simples onde $p=1$. Para a descrição do método veja a Seção 4. Na Seção 3 introduzimos várias definições necessárias para o melhor entendimento da metodologia apresentada aqui. Na Seção 5 exemplificamos a metodologia com a utilização de determinados filtros lineares que serão necessários para analisar o caso $p>1$. Na Seção 6 apresentamos as propriedades estatísticas dos estimadores obtidos através deste método.

\section{Definiçōes gerais.}

Nesta Seção apresentamos algumas definições básicas necessárias para o entendimento das Seções subsequentes.

Utilizamos a notação usual para iterações de aplicações

$$
f^{k}(\alpha)=f\left(f^{k-1}(\alpha)\right), \quad k \in \mathbf{N},
$$

onde $f^{1}(\alpha)=f(\alpha)$ e chamamos $f^{k}(\alpha)$ o k-ésimo iterado da aplicação $f(\cdot)$ no valor $\alpha$.

Desejamos descobrir um método que possibilite encontrar as freqüências através da iteração de uma aplicação $f(\cdot)$. A função $f(\cdot)$ será denotada por $\rho($.$) na Seção 4. Nossa análise do problema será$ baseada nas propriedades geométricas dos gráficos de $f(\cdot)$ e sua derivada nos pontos fixos.

DEFINIÇÃo 3.1: Seja $f$ uma função diferenciável definida de um intervalo $[a, b]$ em si mesmo. Um ponto fixo para a função $f$ é um valor $\alpha^{*}$ tal que $f\left(\alpha^{*}\right)=\alpha^{*}$. 
DEFINIÇÃo 3.2: Um valor $\alpha^{*}$ é dito ser um ponto fixo atrator da aplicação $f(\alpha)$ se $\alpha^{*}$ é um ponto fixo e $\left|f^{\prime}\left(\alpha^{*}\right)\right|<1$. Ele é dito ser um ponto fixo repulsor se $\alpha^{*}$ é um ponto fixo e $\left|f^{\prime}\left(\alpha^{*}\right)\right|>1$.

O conjunto $\left\{\alpha_{0}, f\left(\alpha_{0}\right), f^{2}\left(\alpha_{0}\right), \ldots, f^{k}\left(\alpha_{0}\right), \ldots\right\}$ ou $\left\{\alpha_{0}, \alpha_{1}, \ldots\right.$, $\left.\alpha_{k}, \ldots\right\}$ é chamado a órbita do ponto $\alpha_{0}$.

Propriedade dos pontos atratores: Um ponto fixo atrator $\alpha^{*}$ tcm a propriedade de que pontos próximos dos dois lados de $\alpha^{*}$ são atraídos para $\alpha^{*}$ através de iteraçōes da $f$, isto é,

$$
\lim _{k \rightarrow \infty} f^{k}(\alpha)=\alpha^{*} \text { para todo } \alpha \text { próximo a } \alpha^{*} .
$$

Observamos que esta é uma propriedade local e que não significa necessariamente que $\alpha^{*}$ é um ponto fixo atrator global, isto é, que para quase todo ponto $\alpha_{0} \in(-1,1)$, os iterados $\alpha_{k}$ de $\alpha_{0}$, convergirão para $\alpha^{*}$.

Propriedade dos Pontos Repulsores: Um ponto fixo rcpulsor $\alpha^{*}$ tem a propriedade de que pontos próximos dos dois lados de $\alpha^{*}$ são repelidos de $\alpha^{*}$ através de iteraçõcs de $f$.

$\mathrm{Na}$ prática, pontos fixos repulsores não são observáveis mas pontos fixos atratores podem ser detectados através de altas iterações da aplicação.

4. Método da contração na detecção de freqüências.

O Método da Contração na Deteç̧ão de F́reqüências pode ser mais facilmente descrito no caso em que no modelo temos apenas uma única senóide com freqüência $\omega_{1}$.

Considere o processo estocástico $\left\{Z_{t}\right\}_{t \in \mathbf{Z}}$ dado por

$$
Z_{t}=Y_{t}+\xi_{t}=A \cos \left(\omega_{1} t+\phi\right)+\xi_{t}, \quad t \in \mathbf{Z},
$$

onde $A>0$ e $\omega_{1} \in(0, \pi]$ são constantes, $\phi$ é uma variável aleatória uniformemente distribuída em $(-\pi, \pi]$, isto é, $\phi \sim \mathcal{U}((-\pi, \pi])$ e $\left\{\xi_{t}\right\}_{t \in \mathbf{Z}}$ é um processo estocástico estacionário e ergódico com média zero, independente da fase $\phi$, com função de distribuição espectral $F_{\xi}(\omega)$ contínua em $\omega_{1}$. 
Considere $\left\{\mathcal{L}_{\alpha}(\cdot)\right\}_{\alpha \in \Theta}$ uma família paramétrica de filtros lineares e invariantes no tempo indexados por $\alpha$, onde $\alpha \in \Theta=[\underline{\alpha}, \bar{\alpha}]$ com constantes $\underline{\alpha}$ e $\bar{\alpha}$ tais que $-1<\underline{\alpha}<\cos \left(\omega_{1}\right)<\bar{\alpha}<1$. A função de transferência de $\mathcal{L}_{\alpha}(\cdot)$, denotada por $H(\omega ; \alpha)$, é definida por

$$
H(\omega ; \alpha)=\sum_{j \in \mathbf{Z}} h_{j}(\alpha) e^{-i j \omega},
$$

onde $i=\sqrt{-1}$ e $\left\{h_{j}(\alpha)\right\}_{j \in Z}$ é sua correspondente função de resposta a impulso.

Denotamos por $\left\{Z_{t}(\alpha)\right\}_{t \in \mathbf{Z}}$ o processo filtrado obtido a partir da aplicação do filtro $\mathcal{L}_{\alpha}(\cdot)$ ao processo original e definido por

$$
Z_{t}(\alpha)=\sum_{j \in Z} h_{j}(\alpha) Z_{t-j}=\sum_{j \in \mathrm{Z}} h_{j}(\alpha) Y_{t-j}+\sum_{j \in \mathrm{Z}} h_{j}(\alpha) \xi_{t-j}=Y_{t}(\alpha)+\xi_{t}(\alpha) .
$$

A função de autocorrelação de primeira ordem do processo filtrado $\left\{Z_{t}(\alpha)\right\}_{t \in \mathbf{Z}}$, obtida a partir da representação espectral da função de autocorrelação, é dada por

$$
\begin{aligned}
\rho(\alpha) & =\frac{E\left[Z_{t}(\alpha) Z_{t+1}(\alpha)\right]}{E\left[Z_{t}^{2}(\alpha)\right]}= \\
& =\frac{\frac{A^{2}}{2}\left|H\left(\omega_{1} ; \alpha\right)\right|^{2} \cos \left(\omega_{1}\right)+\int_{-\pi}^{\pi}|H(\omega ; \alpha)|^{2} \cos (\omega) d F_{\xi}(\omega)}{\frac{A^{2}}{2}\left|H\left(\omega_{1} ; \alpha\right)\right|^{2}+\int_{-\pi}^{\pi}|H(\omega ; \alpha)|^{2} d F_{\xi}(\omega)}
\end{aligned}
$$

quando a função de resposta a impulso do filtro $\mathcal{L}_{\alpha}(\cdot)$ tem valor real ou

$$
\begin{aligned}
& \rho(\alpha)=\frac{\mathcal{R}\left\{E\left[Z_{t}(\alpha) \overline{Z_{t+1}(\alpha)}\right]\right\}}{E\left[\left|Z_{t}(\alpha)\right|^{2}\right]}= \\
& =\frac{\frac{A^{2}}{2}\left(\left|H\left(\omega_{1} ; \alpha\right)\right|^{2}+\left|H\left(-\omega_{1} ; \alpha\right)\right|^{2}\right) \cos \left(\omega_{1}\right)+\int_{-\pi}^{\pi}|H(\omega ; \alpha)|^{2} \cos (\omega) d l F_{\xi}(\omega)}{\frac{\Lambda^{2}}{2}\left(\left|H\left(\omega_{1} ; \alpha\right)\right|^{2}+\left|H\left(-\omega_{1} ; \alpha\right)\right|^{2}\right)+\int_{-\pi}^{\pi}|H(\omega ; \alpha)|^{2} d F_{\xi}(\omega)},
\end{aligned}
$$

quando a função de resposta a impulso do filtro $\mathcal{L}_{\alpha}(\cdot)$ tem valor complexo. Nas duas expressões (4.2) e (4.3) $\frac{A^{2}}{2}$ é a variância do sinal 
$\left\{Y_{t}\right\}_{t \in \mathbf{Z}}$. Aqui, e no restante deste artigo, $\mathcal{R}\{z\}$ denota a parte real do número complexo $z$ e $\bar{z}$ denota o conjugado complexo de $z$.

Para simplificar a exposição do Método da Contração na Detecção de Freqüências vamos, a partir deste ponto, fazer uma análise completa quando $p=1$, isto é, o modelo (4.1)

freqüência. Neste caso, é conveniente utilizar a família de filtros paramétricos $\mathcal{L}_{\alpha}(\cdot)$ constituída pelos filtros alfa, definidos a seguir. Nesta situação, a aplicação $\rho(\alpha)$, definida através da expressão (4.2), nos possibilitará obter a freqüência $\omega_{1}$ a ser estimada como o arco cujo coseno é o ponto fixo atrator de $\rho(\cdot)$.

\subsection{O filtro alfa.}

Apresentamos agora uma reformulação do resultado de He e Ke$\operatorname{dem}(1989)$ onde foi analisado o caso $p=1$.

DEFINIÇÃo 4.1: O filtro alfa aplicado a um processo $\left\{Z_{t}\right\}_{t \in \mathbf{Z}}$ é definido pela seguinte transformação linear e invariante no tempo, para todo $\alpha \in(-$

$$
Z_{t}(\alpha)=Z_{t}+\alpha Z_{t-1}(\alpha)
$$

com função ganho quadrada igual a

$$
|H(\omega ; \alpha)|^{2}=\frac{1}{1-2 \alpha \cos () \quad 2},
$$

para todo $\omega \in(-\pi$,

de primeira ordem é dada por

$$
\rho(\alpha)=\frac{E\left[Z_{t}(\alpha) Z_{t+1}(\alpha)\right]}{E\left[Z_{t}^{2}(\alpha)\right]}=\frac{\sum_{j=1}^{p} \frac{A_{j}^{2}}{2} \frac{\cos \left(\omega_{j}\right)}{1-2 \alpha \cos \left(\omega_{j}\right)+\alpha^{2}}+\sigma_{\xi}^{2} \frac{\alpha}{1-\alpha^{2}}}{\sum_{l=1}^{p} \frac{A_{l}^{2}}{2} \frac{1}{1-2 \alpha \cos \left(\omega_{l}\right)+\alpha^{2}}+\sigma_{\xi}^{2} \frac{1}{1-\alpha^{2}}} .
$$

Consideramos agora algumas propriedades da aplicação $\rho$, definida pela expressão (4.5) existência de uma aplicação de contração.

Proposição 4.1: A aplicação $\rho(\alpha)$, dada pela expressão (4 $p=1$, é uma aplicação do intervalo $[-1,1]$ nele mesmo.

A prova desta proposição pode ser encontrada em Lopes (1991). 
Uma longa elaboração algébrica permite mostrar que

$$
\rho^{\prime}(\alpha)=\frac{\frac{A^{2}}{2} \sigma_{\xi}^{2}\left[\frac{1-8 \alpha \cos \left(\omega_{1}\right)+4 \alpha^{2}+2 \alpha^{2} \cos ^{2}\left(\omega_{1}\right)-\alpha^{4}+2 \cos ^{2}\left(\omega_{1}\right)}{\left(1-2 \alpha \cos \left(\omega_{1}\right)+\alpha^{2}\right)^{2}}\right]+\left(\sigma_{\xi}^{2}\right)^{2}}{\left[\frac{A^{2}}{2} \frac{1-\alpha^{2}}{1-2 \alpha \cos \left(\omega_{1}\right)+\alpha^{2}}+\sigma_{\xi}^{2}\right]^{2}} .
$$

Observe que $\rho(-1)=-1$ e $\rho(+1)=+1 \operatorname{mas} \rho^{\prime}(-1)>1 \mathrm{e}$ $\rho^{\prime}(+1)>1$, pois usando a expressão (4.6) acima, temos

$$
\rho^{\prime}(-1)=\frac{\frac{A^{2}}{2}+\sigma_{\xi}^{2}}{\sigma_{\xi}^{2}}=\rho^{\prime}(+1) .
$$

Portanto, -1 e +1 são pontos fixos repulsores da aplicação $\rho(\cdot)$ dada pela expressão (4.5) $\operatorname{com} p=1$, conforme terminologia da Seção 3.

É fácil ver que $\alpha^{*}=\cos \left(\omega_{1}\right)$ é o único ponto fixo em $(-1,1)$ para a aplicação $\rho(\cdot)$ dada pela expressão (4.5) com $p=1$, isto é, $\rho(\alpha)=\alpha \Leftrightarrow \alpha=\cos \left(\omega_{1}\right)=\alpha^{*}$.

Novamente, usando a expressão (4.6) pode-se obter que a derivada de $\rho(\cdot)$ no ponto fixo $\alpha^{*}$ é dada por

$$
\rho^{\prime}\left(\alpha^{*}\right)=\frac{\sigma_{\xi}^{2}}{\frac{A^{2}}{2}+\sigma_{\xi}^{2}} .
$$

Note que este número é estritamente menor do que 1 . Neste caso, o ponto fixo $\alpha^{*}$ é um atrator (observe o gráfico da aplicação $\rho(\cdot)$ em $[-1,1]$ na Figura 1$)$. As propriedades descritas na Seção 3 para a aplicação $f=\rho$ permitem concluir que, para qualquer ponto inicial $\alpha_{0} \in(-1,1)$,

$$
\lim _{k \rightarrow \infty} \rho^{k}\left(\alpha_{0}\right)=\lim _{k \rightarrow \infty} \alpha_{k}=\cos \left(\omega_{1}\right) .
$$

Observe o gráfico de $\rho^{10}(\alpha)$ na Figura 3. Neste caso, como podese ver, qualquer ponto $\alpha_{0}$, iterado $k=10$ vezes está praticamente em cima do valor $\cos \left(\omega_{1}\right)=\alpha^{*}$. Sendo assim, o caso $p=1$ fica, em princípio, totalmente resolvido. É claro que é necessário usar a aplicação $\hat{\rho}(\cdot)$ (um estimador de $\rho(\cdot)$ ) baseada em alguma estimativa da função de autocorrelação de primeira ordem. Nas simulaçōes presentes neste 


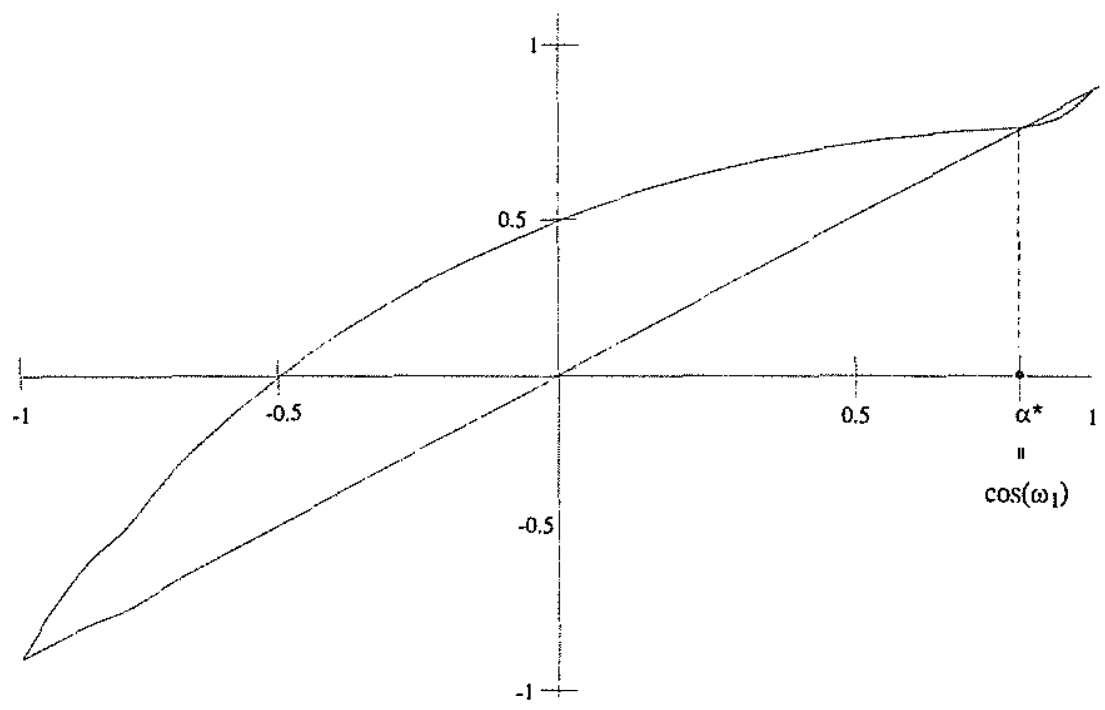

Figura 1.

O ponto fixo atrator $\alpha^{*}=\cos \left(\omega_{1}\right)$ de $\rho(\alpha)$ obtido a partir do filtro alfa, quando $p=1$.

artigo, como aplicação $\hat{\rho}(\cdot)$ consideramos aquela obtida a partir da função de autocorrelação amostral de primeira ordem baseada numa série temporal de tamanho $N$, isto é,

$$
\hat{\rho}(\alpha)=\frac{\frac{1}{N} \sum_{j=1}^{N-1}\left[Z_{j}(\alpha)-\overline{Z(\alpha)}\right]\left[Z_{j+1}(\alpha)-\overline{Z(\alpha)}\right]}{\frac{1}{N} \sum_{j=1}^{N}\left[Z_{j}(\alpha)-\overline{Z(\alpha)}\right]^{2}}
$$

no caso do filtro alfa. A notação $\overline{Z(\alpha)}$, na expressão acima, indica valor médio da série temporal filtrada $\left\{Z_{t}(\alpha)\right\}_{t=1}^{N}$.

O método funciona, mesmo quando em vez de $\rho(\alpha)$ utilizamos $\hat{\rho}(\alpha)$ a expressão estimada da aplicação $\rho(\cdot)$, devido a consistência forte que será demonstrada posteriormente na Seção 6. Sendo assim, o valor estimado $\hat{\alpha}^{*}$ será ponto fixo da aplicação $\hat{\rho}(\cdot)$. 
Análise espectral de sćries

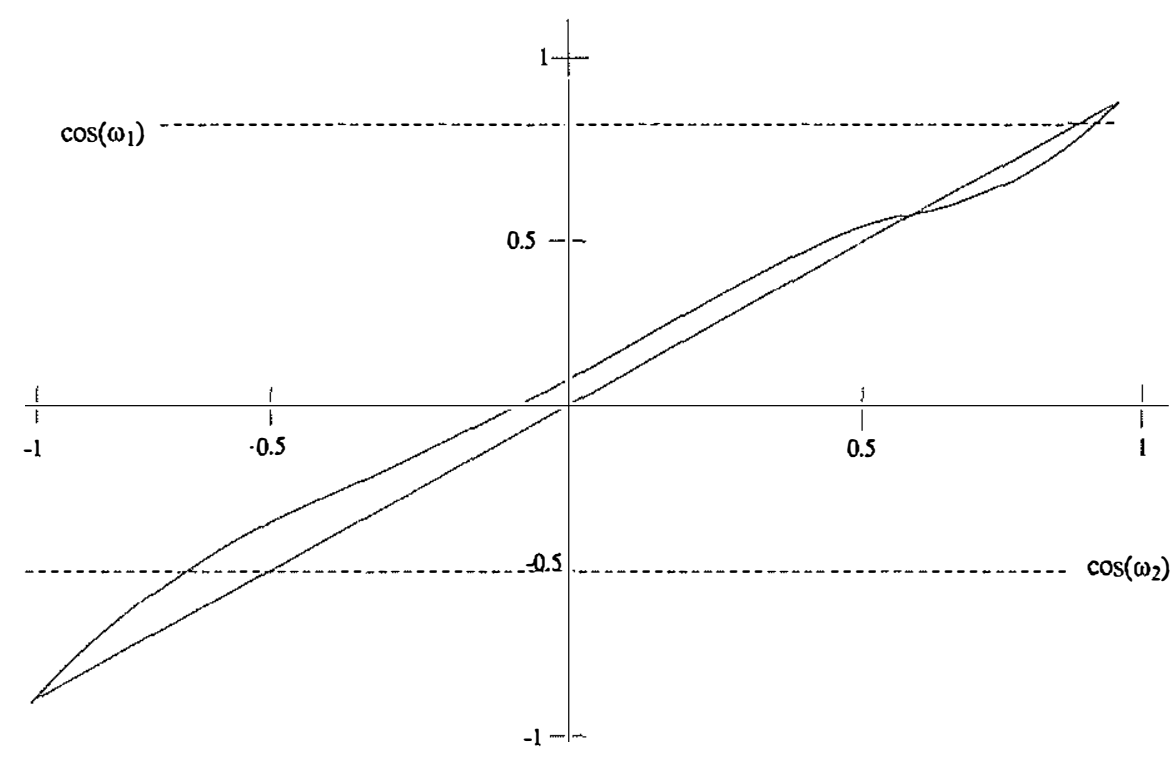

Figura 2.

A aplicação $\rho(\alpha)$ obtida a partir do filtro alfa, quando $p=2$. $\omega_{1}=0.56$ e $\omega_{2}=2.1$ onde $\cos \left(\omega_{1}\right)=0.8472$ e $\cos \left(\omega_{2}\right)=-0.5048$.

Para o caso $p>1$, no entanto, o filtro alfa, através da expressão (4.5) não permitirá a obtenção dos cosenos das freqüências, como pode ser observado na Figura 2, caso $p=2$. Isto porque os cosenos das freqüências não são pontos fixos atratores da aplicação $\rho(\cdot)$. Para obtermos as freqüências $\omega_{j}, 1 \leq j \leq p$, teremos que encontrar outros filtros que definirão outras aplicações $\rho(\cdot)$ que possuirão vários pontos fixos que serão os cosenos das freqüências a serem estimadas. Neste caso, necessitamos introduzir o filtro complexo.

Na literatura da Análise Numérica, o procedimento iterativo utilizado aqui, é conhecido como método iterativo para detectar ponto fixo. Veja, por exemplo, Stoer e Bulirsch (1980)) no caso em que é usado para encontrar raízes da equação $f(x)=x$, através do Método 


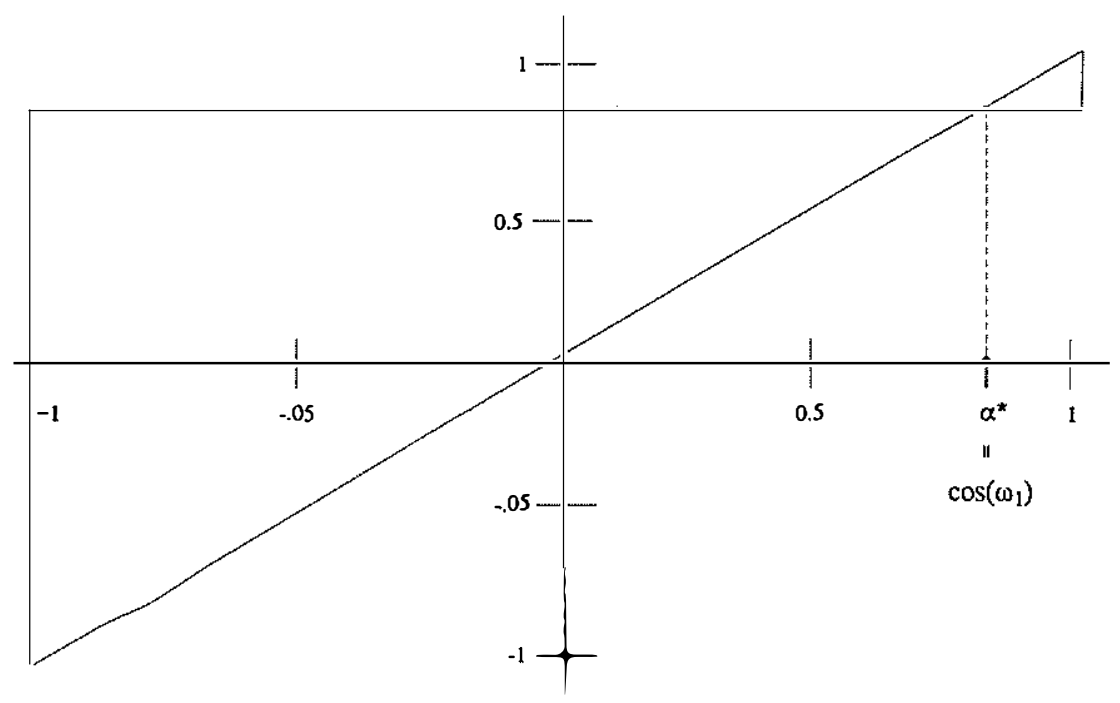

Figura 3.

A aplicação $\rho^{10}(\alpha)$ obtida a partir do filtro alfa quando $p=1$. $\alpha^{*}=\cos \left(\omega_{1}\right)=\cos (0.56)=0.8472$.

de Newton.

$\mathrm{Na}$ Seção 6 estabelecemos que

$$
\hat{\theta}=\operatorname{arc} \cos \left(\hat{\alpha}^{*}\right)=\operatorname{arc} \cos \left(\hat{\rho}\left(\hat{\alpha}^{*}\right)\right)
$$

é o estimador fortemente consistente de $\omega_{1}$. O problema de consistência deste estimador quando $\hat{\rho}(\alpha)$ é função de autocorrelação amostral de primeira ordem foi analisado por Lopes e Kedem (1994) (para o caso em que a família paramétrica $C_{\alpha}(\cdot)$ é formada pelos filtros complexos) e, posteriormente, por Li e Kedem (1993) em outros casos.

\section{O filtro complexo.}

Nesta Seção voltamos a considerar o modelo geral dado pela expressão (1.1) já que a intenção é agora estimar as freqüências $\omega_{j}$, para $1 \leq j \leq p$. 
O material apresentado nesta Seção é um resumo de parte da Seção 1 de Lopes (1991).

DEFINIÇÃo 5.1: O filtro complexo aplicado a um processo $\left\{Z_{t}\right\}_{t \in \mathbf{Z}}$ é definido pela transformação

$$
Z_{t}(\alpha, M)=\left(1+e^{i \theta(\alpha)} \mathcal{B}\right)^{M} Z_{t}
$$

onde $M$ é um número inteiro positivo, $\alpha \in(-1,1), \theta(\alpha) \in(-\pi, \pi)$, $\mathcal{B}$ é o operador defasagem $\mathcal{B} Z_{t}=Z_{t-1}$ e onde $\theta(\alpha)=\arccos (\alpha)$.

Claramente a expressão (5.1) pode ser reescrita como

$$
\begin{aligned}
Z_{t}(\alpha, M)= & \sum_{n=0}^{M}\left(\begin{array}{c}
M \\
n
\end{array}\right) e^{i \theta(\alpha) n} Z_{t-n}, \\
& \text { para } t \in \mathbf{Z}, \quad-\pi<\theta(\alpha)<\pi \text { e } M \in \mathbf{N}-\{0\} .
\end{aligned}
$$

A função de resposta a impulso para este filtro é dada por

$$
h_{j}(\alpha, M)= \begin{cases}\left(\begin{array}{c}
M \\
j
\end{array}\right) e^{i \theta(\alpha) j}, & \text { para } 0 \leq j \leq M, \\
0, & \text { caso contrário. }\end{cases}
$$

A correspondente função ganho quadrada é igual a

$$
|H(\omega ; \alpha, M)|^{2}=4^{M} \cos ^{2 M}\left(\frac{\omega-\theta(\alpha)}{2}\right)
$$

para $\theta(\alpha), \omega \in(-\pi, \pi]$ e $\alpha \in(-1,1)$. Pode-se mostrar rigorosamente (veja Lopes e Kedem (1994)) que, quando $M$ é grande, a correspondente função de autocorrelação de primeira ordem pode ser escrita como

$$
\begin{gathered}
\rho(\alpha, M)=\frac{\mathcal{R}\left\{E\left[Z_{t}(\alpha, M) \overline{Z_{t+1}(\alpha, M)}\right]\right\}}{E\left|Z_{t}(\alpha, M)\right|^{2}} \approx \\
\frac{\sum_{j=1}^{p} \frac{A_{j}^{2}}{2}\left[\cos ^{2 M}\left(\frac{\omega_{j}+\theta(\alpha)}{2}\right)+\cos ^{2 M}\left(\frac{\omega_{j}-\theta(\alpha)}{2}\right)\right] \cos \left(\omega_{j}\right)+\left[\frac{\sigma_{\xi}^{2}}{\pi} \int_{-\pi}^{\pi} \cos ^{2 M}\left(\frac{\lambda-\theta(\alpha)}{2}\right) d \lambda\right] \alpha}{\sum_{l=1}^{p} \frac{A_{l}^{2}}{2}\left[\cos ^{2} M\left(\frac{\omega_{l}+\theta(\alpha)}{2}\right)+\cos ^{2 M}\left(\frac{\omega_{l}-\theta(\alpha)}{2}\right)\right]+\frac{\sigma_{\xi}^{2}}{\pi} \int_{-\pi}^{\pi} \cos ^{2 M}\left(\frac{\lambda-\theta(\alpha)}{2}\right) d \lambda}
\end{gathered}
$$


A qui $\mathcal{R}\{z\}$ significa a parte real do número complexo $z$ e $\bar{z}$ representa o conjugado complexo de $z$.

A expressão (5.2) acima parece muito complexa e será substituída pela expressão (5.3), que é mais fácil de ser entendida.

Observamos que a expressão (5.2) mostra que $\rho(\alpha, M)$ é uma média ponderada de $\cos \left(\omega_{j}\right), j \in\{1, \ldots, p\}$, e de $\alpha$. Definimos os pesos $B_{j}(\alpha, M), j \in\{0,1, \ldots, p\}$, da seguinte forma

$$
B_{j}(\alpha, M)=\frac{\frac{\lambda_{j}^{2}}{2}\left[\cos ^{2 M}\left(\frac{\omega_{j}+\theta(\alpha)}{2}\right)+\cos ^{2 M}\left(\frac{\omega_{j}-\theta(\alpha)}{2}\right)\right]}{\sum_{l=1}^{p} \frac{A_{l}^{2}}{2}\left[\cos ^{2} M\left(\frac{\omega_{l}+\theta(\alpha)}{2}\right)+\cos ^{2 M}\left(\frac{\omega_{1}-\theta(\alpha)}{2}\right)\right]+\frac{\sigma_{\xi}^{2}}{\pi} \int_{-\pi}^{\pi} \cos ^{2 M}\left(\frac{\lambda-\theta(\alpha)}{2}\right) d \lambda},
$$

para $j \in\{1,2, \ldots, p\}$ e

$$
B_{0}(\alpha, M)=\frac{\frac{\sigma_{\xi}^{2}}{\pi} \int_{-\pi}^{\pi} \cos ^{2 M}\left(\frac{\lambda-\theta(\alpha)}{2}\right) d \lambda}{\sum_{l=1}^{p} \frac{A_{l}^{2}}{2}\left[\cos ^{2 M}\left(\frac{\omega_{l}+\theta(\alpha)}{2}\right)+\cos ^{2 M}\left(\frac{\omega_{l}-\theta(\alpha)}{2}\right)\right]+\frac{\sigma_{\xi}^{2}}{\pi} \int_{-\pi}^{\pi} \cos ^{2 M}\left(\frac{\lambda-\theta(\alpha)}{2}\right) d \lambda}
$$

Observe que $\sum_{j=0}^{p} B_{j}(\alpha, M)=1$ e, portanto, $\rho(\alpha, M)$ é uma média ponderada envolvendo $\cos \left(\omega_{j}\right), 1 \leq j \leq p$, e $\alpha$ dada por

$$
\rho(\alpha)=\rho(\alpha, M)=\sum_{j=1}^{p} B_{j}(\alpha, M) \cos \left(\omega_{j}\right)+B_{0}(\alpha, M) \alpha
$$

onde os pesos $B_{j}(\alpha, M), 0 \leq j \leq p$, são não-negativos, somam um e dependem de $\alpha$ e $M$. Esta observação é importante na recuperação de todas as freqüências $\omega_{j}, j \in\{1, \ldots, p\}$.

Consideramos agora algumas propriedades da aplicação $\rho$, definida em (5.2), que auxiliarão na prova da existência de uma aplicação de contração.

Proposição 5.1: Para cada $M \in \mathrm{N}-\{0\}$ fixado e $\rho(\alpha)=\rho(\alpha, M)$, dada pela expressão (5.2), a aplicação $\rho(\cdot)$ é uma função do intervalo $[-1,1]$ nele mesmo, como uma função da variável $\alpha$.

A prova desta proposição é similar àquela da Proposição 4.1 considerando agora a expressão (5.2).

Queremos fazer alguns comentários a respeito da aplicação $\rho(\alpha)=$ $\rho(\alpha, M)$ dada pelas expressões (5.2) ou (5.3). 
Resultado principal para o Filtro COMPLEXo: Sc $M I$ é grande c fixo (suponha, por excmplo, $M=30$ ), cntão dado $\alpha_{0} \in(-1,1)$ escolhido ao acaso, $\rho^{k}\left(\alpha_{0}\right)$ estará bem próximo de um ponto fixo $\alpha^{*}$, onde $\alpha^{*}$ é um dos possivivis valores $\cos \left(\omega_{j}\right)$, para $1 \leq j \leq p$, quando $k$ for tomado grandc.

Isto permitirá obter as várias freqüências do modelo (2.1) através de um procedimento iterativo similar ao que foi utilizado no caso $p=1$ com o filtro alfa. Neste caso, $\hat{\rho}^{k}\left(\alpha_{0}\right)$, com $k$ grande, estará estimando um possível $\cos \left(\omega_{j}\right)$.

Vamos tentar explicar de uma maneira heurística a razão pela qual o Resultado Principal é verdadeiro. Para simplificar o raciocínio, suponha que $\alpha_{0}$ está mais próximo de $\cos \left(\omega_{1}\right)$ do que dos demais $\cos \left(\omega_{j}\right)$, para $2 \leq j \leq p$. Dessa forma, é fácil ver que

$$
\lim _{M \rightarrow \infty} \frac{\cos ^{2 M}\left(\frac{\omega_{j}+\theta\left(\alpha_{0}\right)}{2}\right)+\cos ^{2 M}\left(\frac{\omega_{j}-\theta\left(\alpha_{0}\right)}{2}\right)}{\cos ^{2 M}\left(\frac{\omega_{1}-\theta\left(\alpha_{0}\right)}{2}\right)}=0,
$$

para todo $j \in\{2, \ldots, p\} \mathrm{e}$

$$
\lim _{M \rightarrow \infty} \frac{\cos ^{2 M}\left(\frac{\omega_{1}+\theta\left(\alpha_{0}\right)}{2}\right)}{\cos ^{2 M}\left(\frac{\omega_{1}-\theta\left(\alpha_{0}\right)}{2}\right)}=0 .
$$

Lembre que $\theta\left(\alpha_{0}\right)=\arccos \left(\alpha_{0}\right)$. A partir das duas igualdades acima segue-se

$$
\lim _{M \rightarrow \infty} \frac{B_{j}\left(\alpha_{0}, M I\right)}{B_{1}\left(\alpha_{0}, M\right)}=0
$$

para todo $j \in\{2,3, \ldots, p\}$. A expressão acima significa que o peso relativo $B_{1}\left(\alpha_{0}, M\right)$ é muito maior do que os outros $B_{j}\left(\alpha_{0}, M\right)$, se $M$ é grande e $\alpha_{0}$ está próximo de $\omega_{1}$.

$\mathrm{Da}$ expressão (5.3) podemos concluir que, para o valor inicial $\alpha=\alpha_{0}$ próximo a $\cos \left(\omega_{1}\right)$,

$$
\alpha_{1}=\rho\left(\alpha_{0}\right)=\rho(\alpha)=\sum_{j=1}^{p} B_{j}(\alpha, M) \cos \left(\omega_{j}\right)+B_{0}(\alpha, M) \alpha
$$


é, na verdade, uma combinação linear ponderada de $\alpha_{0}$ e dos $\cos \left(\omega_{j}\right)$, para todo $j \in\{1,2, \ldots, p\}$.

Supondo $M$ grande e fixo então, pela igualdade (5.4), concluímos que a média $\alpha_{1}=\rho\left(\alpha_{0}\right)$ tem uma tendência muito forte de estar próxima a $\cos \left(\omega_{1}\right)$ pois o peso $B_{1}\left(\alpha_{0}, M\right)$ é muito maior do que os demais pesos $B_{j}\left(\alpha_{0}, M\right)$, para $j \in\{0,2, \ldots, p\}$. Aplicando o mesmo raciocínio a $\alpha_{2}=\rho\left(\alpha_{1}\right)$, temos que $\alpha_{2}$ deve estar ainda mais próxima de $\cos \left(\omega_{1}\right)$ do que $\alpha_{1}$. Aplicando agora sucessivamente $k$ vezes o raciocínio, concluímos que $\alpha_{k}=\rho^{k}\left(\alpha_{0}\right)=\rho^{k}(\alpha)$ deve estar bastante próximo de $\cos \left(\omega_{1}\right)$, e é um bom estimador para o coseno de uma das freqüências, no caso $\omega_{1}$. De forma análoga, se $\alpha$ estivesse mais próximo de $\cos \left(\omega_{2}\right)$ do que dos demais $\cos \left(\omega_{j}\right)$, para $j \in\{1,3, \ldots, p\}$, então $\rho^{k}(\alpha)$ deveria estar bastante próximo de $\cos \left(\omega_{2}\right)$, e assim por diante.

Como no caso do filtro alfa, aqui também estaremos usando uma estimativa $\hat{\rho}(\cdot, M)=\hat{\rho}(\cdot)$ para a obtenção da aplicação $\rho(\cdot, M)=\rho(\cdot)$. Nas simulações apresentadas nestas notas, utilizamos como estimativa para $\rho(\cdot, M)$ a função de autocorrelação amostral de primeira ordem da série temporal filtrada $\left\{Z_{t}(\alpha, M)\right\}_{t=1}^{N}$ de tamanho $N$. Esta estimativa é dada por

$$
\hat{\rho}(\alpha, M)=\frac{\frac{1}{N} \mathcal{R}\left\{\sum_{j=1}^{N-1}\left[Z_{j}(\alpha, M)-\overline{Z(\alpha, M)}\right]\left[\overline{\left.Z_{j+1}(\alpha, M)-\overline{Z(\alpha, M)}\right]}\right\}\right.}{\frac{1}{N} \sum_{j=1}^{N}\left[Z_{j}(\alpha, M)-\overline{Z(\alpha, M)}\right]\left[\overline{\left.Z_{j}(\alpha, M)-\overline{Z(\alpha, M)}\right]}\right.}
$$

onde a notação $\overline{Z(\alpha, M)}$ indica o valor médio da série temporal filtrada $\left\{Z_{t}(\alpha, M)\right\}_{t=1}^{N}$.

A consistência forte pode ser demonstrada neste caso, e então todo o procedimento acima descrito pode ser totalmente justificado (ver Lopes e Kedem (1994)).

A eficácia deste procedimento pode ser observada através da análise dos gráficos da função $\rho(\cdot)=\rho(\cdot, M)$ na situação em que temos duas e três freqüências (veja as Figuras 4, 5, 6 e 7). Para apreciar corretamente o efeito do acréscimo de $M$ na localização exata das freqüências, observe a Figura 8. Para o valor $M=15$ se obtém uma boa precisão para o valor a ser estimado através da iteração de $\rho(\cdot)$. Observe que a freqüência não é um ponto fixo, mas existe um ponto 
fixo atrator bem próximo a ela. Quando $M \rightarrow \infty$ este ponto fixo atrator tende a coincidir com o coseno da freqüência a ser detectada.

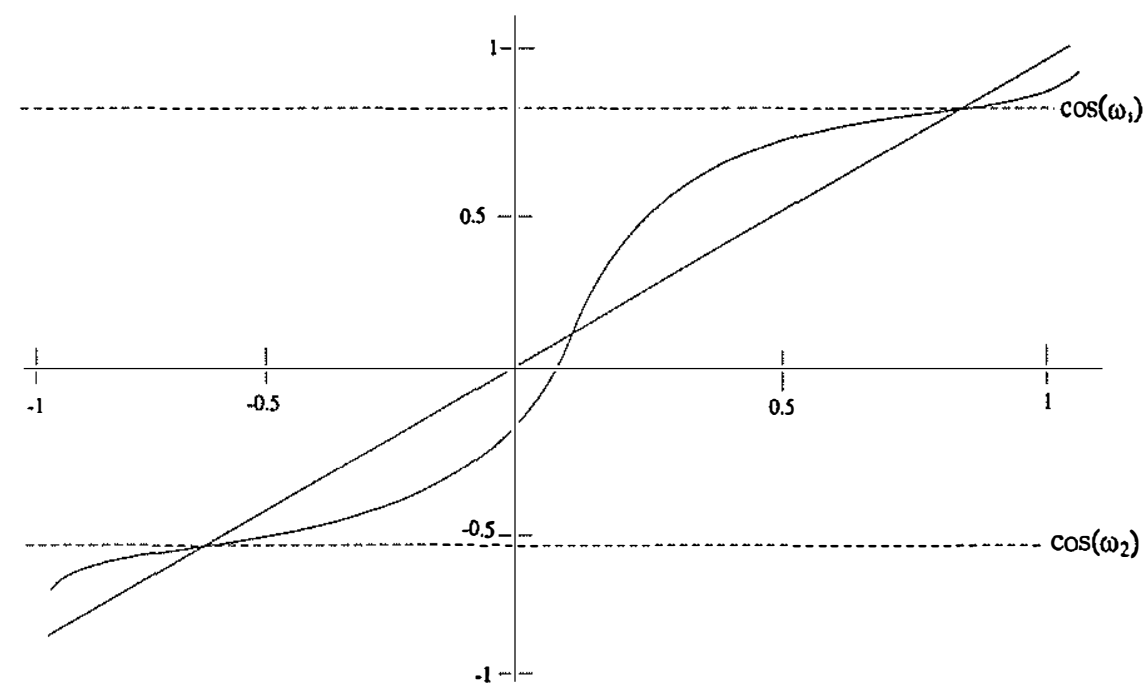

Figura 4.

Pontos Fixos para $\rho(\alpha, 15)$ obtida a partir do filtro complexo com $p=2, A_{1}=A_{2}=\sigma_{\xi}=1.0, \omega_{1}=0.7 \mathrm{e}$ $\omega_{2}=2.2\left(\cos \left(\omega_{1}\right)=0.7684, \cos \left(\omega_{2}\right)=-0.5885\right)$.

Os gráficos que apresentamos em todas as figuras foram feitos para as expressões analíticas $\rho(\cdot)$. O gráfico da respectiva aplicação $\hat{\rho}(\cdot)$ deveria estar bem próximo do gráfico da respectiva aplicação $\rho(\cdot)$. Em conclusão, $\hat{\rho}^{k}\left(\alpha_{0}\right)=\hat{\alpha}_{k}$ é um estimador de alguma freqüência, se $k$ for suficientemente grande.

\section{Propriedades estatísticas dos estimadores $M C$.}

Antes de enunciarmos formalmente o teorema que garante a convergência da seqüência dos estimadores $\hat{\alpha}_{k}$ baseados em uma série 


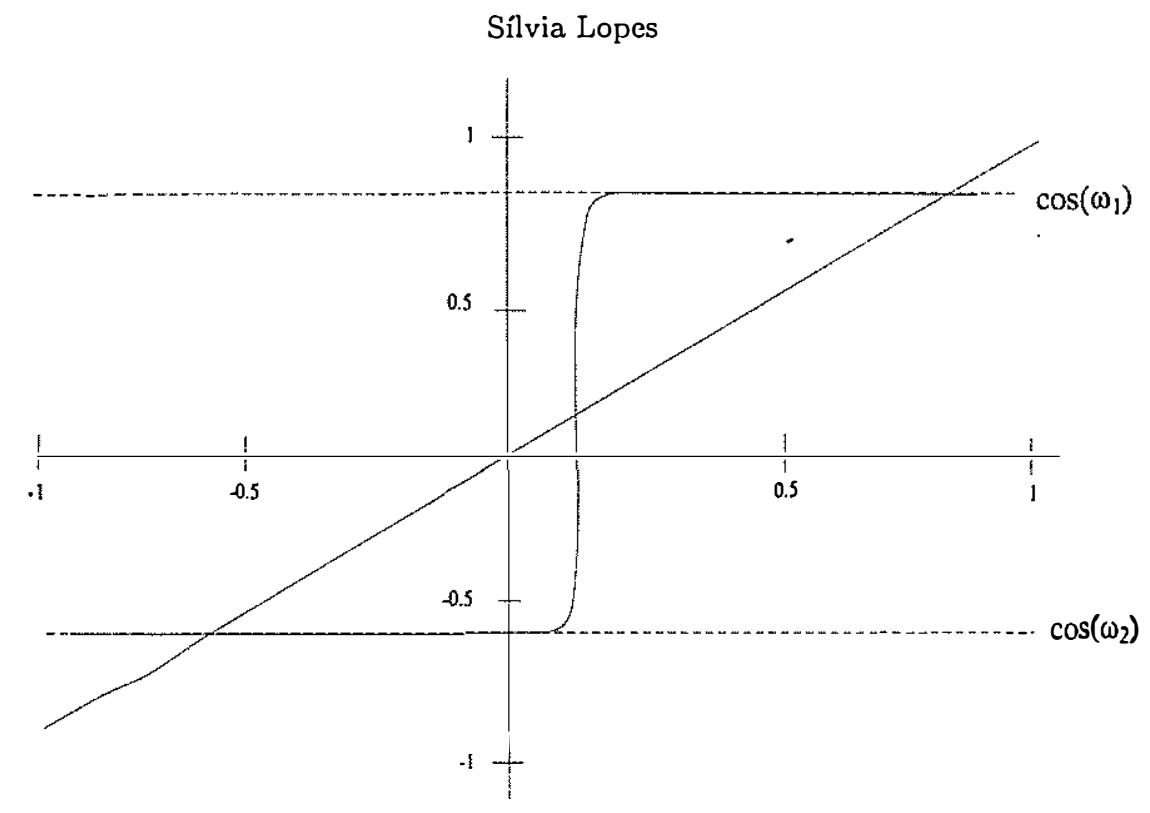

Figura 5.

Pontos Fixos para $\rho(\alpha, 15)$ obtida a partir do filtro complexo com $p=2, A_{1}=A_{2}=\sigma_{\xi}=1.0, \omega_{1}=0.7 \mathrm{e}$ $\omega_{2}=2.2\left(\cos \left(\omega_{1}\right)=0.7684, \cos \left(\omega_{2}\right)=-0.5885\right)$.

temporal filtrada através de filtros complexos, vamos introduzir a seguinte definição.

DEFINIÇÃo 6.1: O procedimento iterativo (1.1) de atualização de parâmetros é dito ser aproximadamente globalmente convergente se para cada $M \in \mathrm{N}-\{0\}$ fixo existe um conjunto $C_{M}$ de medida de Lebesgue total em $[-1,1]$ tal que para qualquer $\alpha_{0} \in C_{M}$ existe o limite

$$
\lim _{k \rightarrow \infty} \rho^{k}\left(\alpha_{0}\right)=\lim _{k \rightarrow \infty} \alpha_{k+1}=\alpha_{M}^{*}
$$

Os possíveis valores $\alpha_{M}^{*}$ são pontos fixos da aplicaçãa $\rho(\cdot)$, dạda pela expressão (5.2), isto é, $\rho\left(\alpha_{M}^{*}\right)=\alpha_{M}^{*}$.

O procedimento iterativo de atualização de parâmetros é considerado com respeito ao filtro com parâmetro fixo $M$ e o valor limite $\alpha_{M}^{*}$ pode depender de $\alpha_{0}$, o valor inicial. Nós exigimos também nesta 


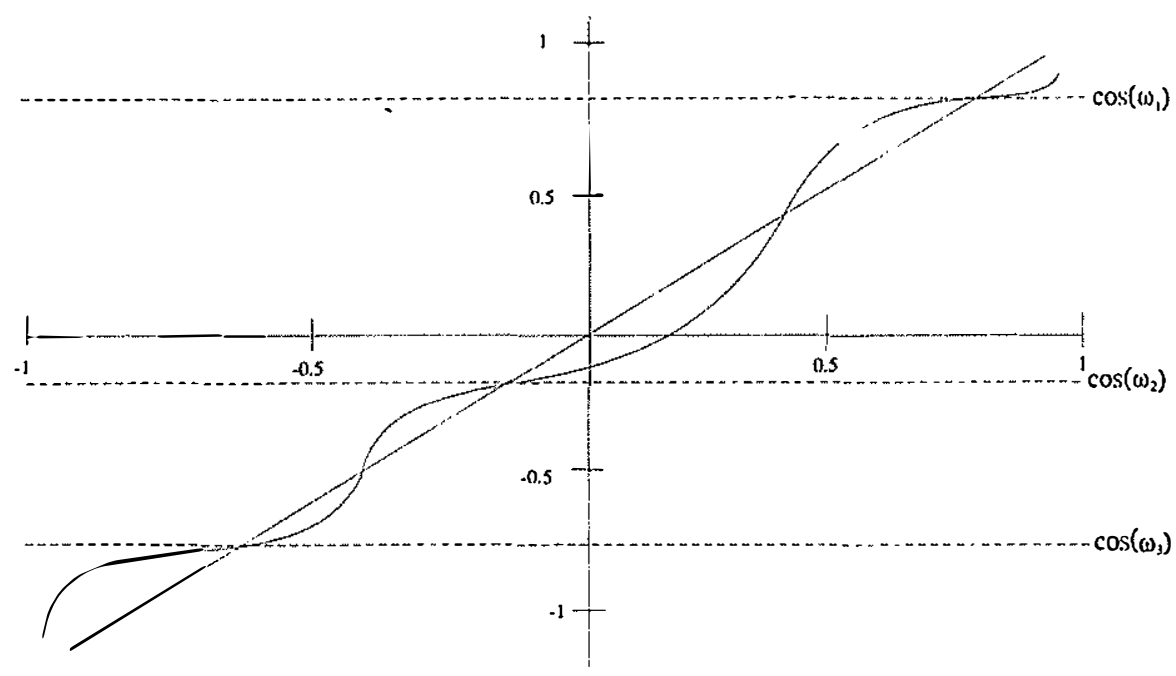

Figura 6.

Pontos Fixos para $\rho(\alpha, 40)$ obtida a partir do filtro complexo com $p=3, A_{1}=A_{2}=A_{3}=\sigma_{\xi}=1.0, \omega_{1}=$ $0.5, \omega_{2}=1.7$ e $\omega_{3}=2.4\left(\cos \left(\omega_{1}\right)=0.8775, \cos \left(\omega_{2}\right)=\right.$ -0.1288 e $\left.\cos \left(\omega_{3}\right)=-0.7373\right)$.

definição que existam $p$ destes possíveis valores $\alpha_{M}^{*}$ e que, para cada um deles, exista $l \in\{1,2, \ldots, p\}$ tal que

$$
\lim _{M \rightarrow \infty} \alpha_{M}^{*}=\cos \left(\omega_{l}\right)
$$

O resultado a seguir expressa matematicamente o que explicamos de maneira heurística na Seção 5 através do Resultado Principal e garante que a obtenção das freqüências do processo (2.1) é feita através dos pontos fixos da aplicação $\rho(\cdot)$, dada pela expressão (5.2). Veja Lopes e Kedem (1994) para a demonstração completa.

Teorema 6.1: Considere o processo estocástico $\left\{Z_{t}\right\}_{t \in \mathbf{Z}}$ como em (2.1) onde o processo $\left\{\xi_{t}\right\}_{t \in \mathbf{Z}}$ é um ruído branco Gaussiano. Seja $\left\{\mathcal{L}_{\theta}(Z)_{t}\right\}_{\theta \in \Theta}=\left\{Z_{t}(\alpha, M)\right\}_{(\alpha, M) \in \Theta}$, onde $\theta=(\alpha, M) \in(-1,1) \times$ 


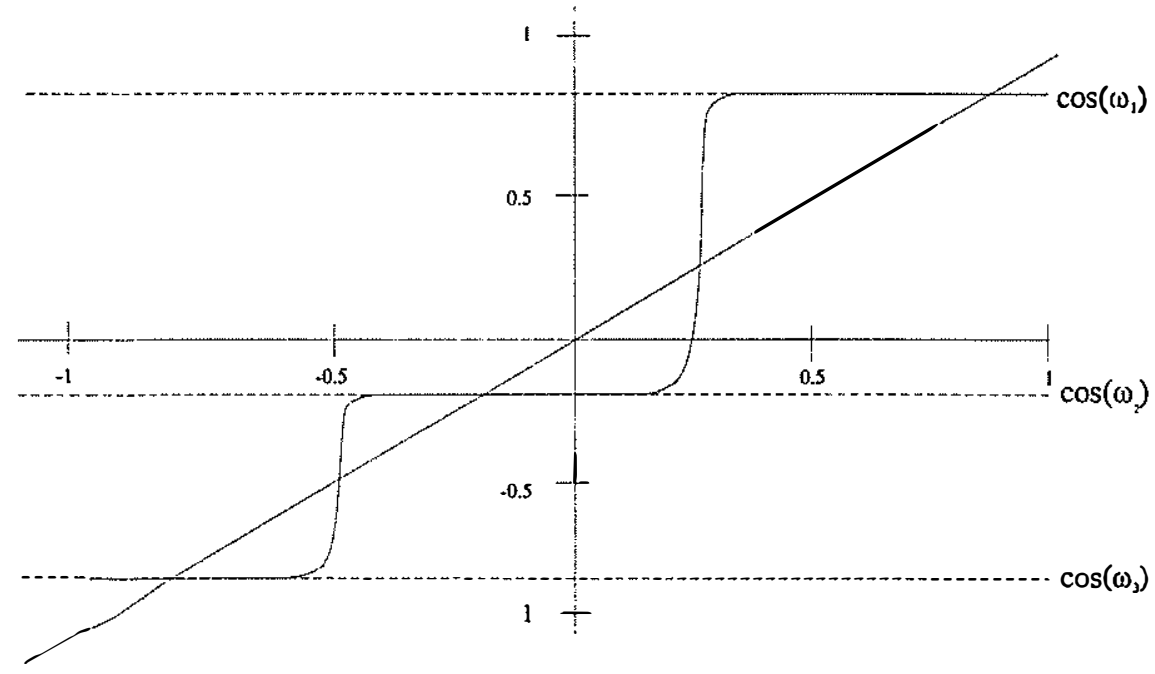

Figura 7.

Pontos Fixos para $\rho^{30}(\alpha, 40)$ obtida a partir do filtro complexo com $p=3, A_{1}=A_{2}=A_{3}=\sigma_{\xi}=1.0, \omega_{1}=$ $0.5, \omega_{2}=1.7$ e $\omega_{3}=2.4\left(\cos \left(\omega_{1}\right)=0.8775, \cos \left(\omega_{2}\right)=\right.$ -0.1288 e $\left.\cos \left(\omega_{3}\right)=-0.7373\right)$.

$\mathrm{N}=\Theta$, a família paramétrica dos filtros complexos. Considere ainda o procedimento iterativo (1.1). Então, a família $\left\{\mathcal{L}_{\theta}(Z)_{t}\right\}_{\theta \in \Theta}$ é aproximadamente globalmente convergente.

Veja as Tabelas 6.1 e 6.2.

\subsection{Ergodicidade do processo estocástico.}

Apresentamos a prova da consistência forte (ou equivalentemente, da ergodicidade), dos estimadores utilizados neste trabalho, apenas para o caso $p=1$. Para o caso geral, veja Lopes e Kedem (1994). Considere $(\Omega, \mathcal{F}, \mathrm{P})$ um espaço de probabilidade onde $\Omega$ é o espaço amostral, $\mathcal{F}$ é a $\sigma$-álgebra de Borel e $\mathrm{P}$ é a função de probabilidade definida em $\Omega$. Considere a transformação $T$ definida sobre o espaço $\Omega$ nele mesmo. 
Análise espectral de séries

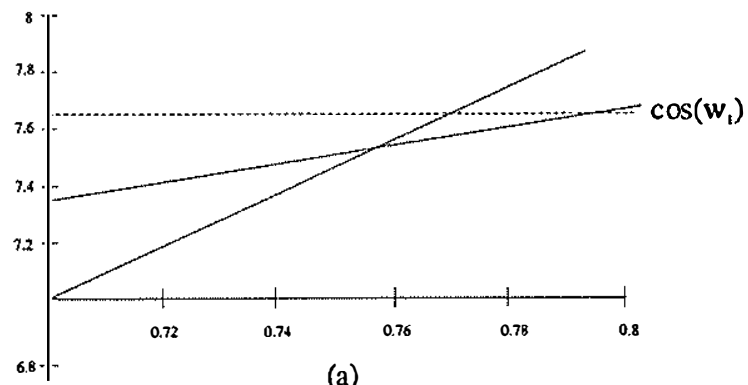

(a)
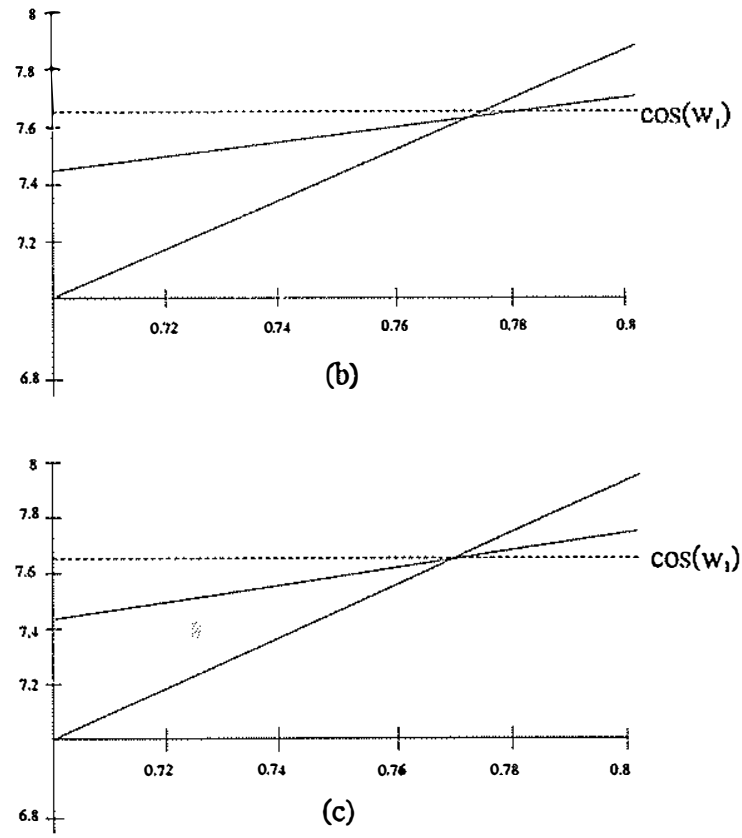

Figura 8.

$\rho(\alpha, M)$ obtida a partir do filtro complexo para $p=2$ numa vizinhança do coseno da freqüência $\omega_{1}=0.7\left(\cos \left(\omega_{1}\right)=\right.$ 0.7684). O gráfico da função constante $y=\cos \left(\omega_{1}\right)$ e da reta da diagonal também estão plotados. (a) $M=8$; (b) $M=11$; (c) $M=15$. 
Tabela 6.1.

Estimativa da Freqüência $\omega_{j}, j=1,2$, a partir do filtro complexo. $p=2, \omega_{1}=0.8, \omega_{2}=2.2, N=3000 \mathrm{e}$ $S N R=20 \log _{10}\left(\frac{\text { desv.pad. sinal }}{\text { desv.pad. rúdo }}\right) d B$. Número de Iteraçōes $=8$.

\begin{tabular}{cccccccc}
\hline Freqüência Estimada & $A_{1}$ & $A_{2}$ & $\sigma_{\xi}$ & $S N R(d B)$ & $M$ & $\Theta_{0}$ & $\omega_{j}$ \\
\hline \multirow{4}{*}{$\omega_{1}$} & 1.0 & 1.0 & 1.0 & 0 & 15 & 0.5 & 0.84721 \\
& 1.0 & 1.0 & 1.0 & 0 & 20 & 0.5 & 0.83152 \\
& 1.0 & 1.0 & 1.0 & 0 & 25 & 0.5 & 0.82310 \\
& 1.0 & 1.0 & 1.0 & 0 & 30 & 0.5 & 0.81827 \\
& 1.0 & 1.0 & 1.0 & 0 & 34 & 0.5 & 0.81557 \\
\hline \multirow{3}{*}{$\omega_{2}$} & 1.0 & 1.0 & 1.0 & 0 & 15 & 1.9 & 2.17078 \\
& 1.0 & 1.0 & 1.0 & 0 & 20 & 1.9 & 2.18241 \\
& 1.0 & 1.0 & 1.0 & 0 & 25 & 1.9 & 2.18823 \\
& 1.0 & 1.0 & 1.0 & 0 & 30 & 1.9 & 2.19164 \\
& 1.0 & 1.0 & 1.0 & 0 & 34 & 1.9 & 2.19337 \\
& 2.0 & 1.0 & 1.0 & 4 & 15 & 1.9 & 2.19260 \\
& 2.0 & 1.0 & 0.5 & 10 & 15 & 1.9 & 2.19786 \\
\hline
\end{tabular}

DeFInIÇÃo 6.2: Dizemos que $\mathbf{P}$ é uma medida invariante sobre $T$ se $\mathbf{P}\left(T^{-1}(B)\right)=\mathbf{P}(B)$, para todo boreliano $B \in \mathcal{F}$.

DefinIÇão 6.3: Dizemos que $\mathbf{P}$ é ergódica sobre $T$ se, para todo boreliano $B \in \mathcal{F}$ tal que $T^{-1}(B)=B$, temos $\mathbf{P}(B)=0$ ou $\mathbf{P}(B)=1$.

Um resultado muito importante é o Teorema Ergódico de Birkhoff (veja Cornfeld, Fomin e Sinai (1982)) que enunciamos a seguir.

Teorema Ergódico de Birkhoff 6.2: Considere $V$ uma variável aleatória integrável em $\Omega, \mathbf{P}$ é uma medida de probabilidade invariante em $\Omega$ e $T$ é uma transformação mensurável em $\Omega$. Seja $\mathcal{G}$ a menor $\sigma$-álgebra de conjuntos em $\mathcal{F}$ com respeito a qual todas as variáveis aleatórias $W$ com $W\left(T^{t}(\omega)\right)=W(\omega)$ para $\mathbf{P}$-quase todo $\omega$ e para $t>0$ são mensuráveis. Então,

$$
\lim _{N \rightarrow \infty} \frac{1}{N} \sum_{t=0}^{N-1} V\left(T^{t}(\omega)\right)=E(V / \mathcal{G})(\omega) \mathbf{P} \text { - quase certo. }
$$

Quando $\mathbf{P}$ é ergódica (isto é, $\mathcal{G}$ é trivial) então $E(V / \mathcal{G})$ se reduz a $E(V)=$ constante e o resultado acima essencialmente diz que para 
Tabela 6.2.

Estimativa da Freqüência $\omega_{j}, j=1,2$, a partir do filtro complexo. $p=2, \omega_{1}=0.5, \omega_{2}=2.5, N=3000 \mathrm{e}$ $S N R=20 \log _{10}\left(\frac{\text { desv.pad.sinal }}{\text { desv.pad. ruído }}\right) d B$. Número de Iterações $=8$.

\begin{tabular}{cccccccc}
\hline Frequiência Estimada & $A_{1}$ & $A_{2}$ & $\sigma_{\xi}$ & $S N R(d B)$ & $M$ & $\Theta_{o}$ & $\omega_{j}$ \\
\hline \multirow{3}{*}{$\omega_{1}$} & 1.0 & 1.0 & 1.0 & 0 & 15 & 0.3 & 0.56217 \\
& 1.0 & 1.0 & 1.0 & 0 & 20 & 0.3 & 0.53882 \\
& 1.0 & 1.0 & 1.0 & 0 & 25 & 0.3 & 0.52659 \\
& 1.0 & 1.0 & 1.0 & 0 & 30 & 0.3 & 0.51954 \\
& 1.0 & 1.0 & 1.0 & 0 & 34 & 0.3 & 0.51587 \\
& 1.0 & 2.0 & 1.0 & 4 & 15 & 0.1 & 0.51628 \\
\hline \multirow{6}{*}{$\omega_{2}$} & 1.0 & 1.0 & 1.0 & 0 & 15 & 2.9 & 2.46535 \\
& 1.0 & 1.0 & 1.0 & 0 & 20 & 2.9 & 2.47876 \\
& 1.0 & 1.0 & 1.0 & 0 & 25 & 2.9 & 2.48631 \\
& 1.0 & 1.0 & 1.0 & 0 & 30 & 2.9 & 2.49077 \\
& 1.0 & 1.0 & 1.0 & 0 & 34 & 2.9 & 2.49303 \\
& 0.5 & 1.0 & 1.0 & -2 & 15 & 3.1 & 2.37030 \\
& 2.0 & 1.0 & 1.0 & 4 & 15 & 2.8 & 2.49065 \\
\hline
\end{tabular}

típicas trajetórias com respeito a $\mathrm{P}$, a média temporal de $V$ converge para a média espacial de $V$.

Em termos de processos estocásticos, nós estamos considerando na terminologia acima o processo estacionário $Y_{t}(\omega)=V\left(T^{t}(\omega)\right)$, $\omega \in \Omega$ e $t \in \mathbf{Z}$. Esta é a maneira usual de transferir resultados de transformações com medidas invariantes para processos estocásticos (veja Lamperti (1977), Capítulo 5, para maiores detalhes). Basicamente, temos que considerar no espaço $\Omega^{\mathrm{N}}$, a medida produto gerada por $\mathrm{P}$ em $\Omega$ e a definição acima do processo estocástico $Y_{t}$. Nós observamos aqui que $\mathbf{P}$ será a medida produto no caso em que tivermos coordenadas independentes e identicamente distribuídas.

ObSERVAÇÃo 6.1: Suponha que $\int V(\omega) \mathbf{P}(d \omega)=0$. Então, neste caso, se a probabilidade é ergódica, a autocovariância de ordem $k$

$$
\int V(\omega) V\left(T^{k}(\omega)\right) \mathbf{P}(d \omega)
$$


pode ser obtida como o limite quase certo da média

$$
\lim _{N \rightarrow \infty} \frac{1}{N}_{t=0}^{N-k}
$$

Neste caso, podemos dizer que a autocovariância amostral de primeira ordem (caso $k=1$ )

estimadores fortemente consistentes.

No nosso caso devemos considerar $\Omega=(-\pi, \pi]$ e para qualquer $\omega \in \Omega$, temos

$$
T(\omega)=\omega+\omega_{1}(\bmod 2 \pi),
$$

onde $\omega_{1}$ é uma constante fixa no intervalo $(-\pi, \pi]$. Agora $\mathbf{P}$ é a medida de Lebesgue normalizada em $(-\pi, \pi]$ e esta probabilidade é claramente invariante para $T$.

É conhecido (veja Cornfeld, Fomin e Sinai (1982), página 64) que, quando $\frac{\omega_{1}}{2 \pi}$ é um número irracional, então $\mathbf{P}$ é ergódica para $T$. OBSERVAÇÃo 6.2: O Teorema Ergódico quando $\frac{\omega_{1}}{2 \pi}$ é irracional, é verdadeiro numa forma mais forte do que o Teorema Ergódico de Birkhoff. De fato, a afirmação acima a respeito das médias temporais é verdadeira, não apenas $\mathbf{P}$-quase certo, mas na verdade para todo $\omega \in(-\pi, \pi]$. A afirmação análoga para os números $\omega_{1}$ quando $\frac{\omega_{1}}{2 \pi}$ é racional não é verdadeira.

Para a situação que temos aqui, vamos denotar os elementos do espaço amostral $\Omega$ por $\phi$. Desta forma teremos uma notação coerente com o visto anteriormente. Consideremos a variável aleatória $V(\omega)=$ $V(\phi)=A \cos (\phi)$. Observe que

$$
\int V(\omega) \mathbf{P}(d \omega)=A \int \cos (\phi) \mathbf{P}(d \phi)=0
$$

Portanto, a hipótese da Observação 6.1 Observe que para qualquer $n \in \mathrm{N}$ e $\phi \in(-\pi, \pi]$, temos que $T^{n}(\phi)=$ $\phi+n \omega_{1}(\bmod 2 \pi)$.

Se $\frac{\omega_{1}}{2 \pi}$ é irracional então podemos aplicar o Teorema Ergódico para a variável aleatória $V(\phi) V\left(T^{k}(\phi)\right)$, já que $\mathbf{P}$ é ergódica (veja a 
Observação 6.1). Desta forma, nós temos um estimador consistente para a autocovariância.

Portanto, pelo Teorema Ergódico segue que

$$
\begin{aligned}
& \lim _{N \rightarrow \infty} \frac{1}{N} \sum_{t=0}^{N-k} V\left(T^{t}(\phi)\right) V\left(T^{t+k}(\phi)\right)= \\
& =\lim _{N \rightarrow \infty} \frac{1}{N} A^{2} \sum_{t=0}^{N-k} \cos \left(\omega_{1} t+\phi\right) \cos \left(\omega_{1}(t+k)+\phi\right)= \\
& =A^{2} \int \cos (\phi) \cos \left(\omega_{1} k+\phi\right) \mathrm{P}(d \phi)= \\
& =A^{2} \int \cos (\phi) \cos \left(T^{k}(\phi)\right) \mathrm{P}(d \phi), \text { para } k \geq 0 .
\end{aligned}
$$

Portanto, para qualquer $\phi \in(-\pi, \pi]$, temos que as autocovariâncias amostrais de ordem $k$ fornecem estimadores fortemente consistentes para a autocovariância de ordem $k$ do processo $\left\{Y_{t}\right\}_{t \in \mathbf{Z}}$. Relembramos que primeiro consideramos o processo estocástico

$$
Y_{t}(\phi)=V\left(T^{t}(\phi)\right)=V\left(\phi+t \omega_{1}\right)=A \cos \left(\omega_{1} t+\phi\right) .
$$

Queremos agora adicionar o processo do ruído branco Gaussiano $\xi_{t}$ ao $Y_{t}$. Portanto, queremos analisar a autocovariância de ordem $k$ para o processo $Z_{t}$ dado por

$$
Z_{t}=Y_{t}+\xi_{t}=V\left(T^{t}(\cdot)\right)+\xi_{t} .
$$

Observamos que, para todo $k \geq 0$,

$$
Z_{t}(\phi) Z_{t+k}(\phi)=Y_{t}(\phi) Y_{t+k}(\phi)+Y_{t}(\phi) \xi_{t+k}+Y_{t+k}(\phi) \xi_{t}+\xi_{t .} \xi_{t+k} .
$$

A correspondente igualdade acima no caso amostral é dada por

$$
\begin{aligned}
\frac{1}{N} \sum_{t=0}^{N-k} Z_{t}(\phi) Z_{t+1}(\phi) & =\frac{1}{N} \sum_{t=0}^{N-k} V\left(T^{t}(\phi)\right) V\left(T^{t+k}(\phi)\right) \\
& +\frac{1}{N} \sum_{t=0}^{N-k} V\left(T^{t}(\phi) \xi_{t+k}+\frac{1}{N} \sum_{t=0}^{N-k} V\left(T^{t+k}(\phi)\right) \xi_{t}\right. \\
& +\frac{1}{N} \sum_{t=0}^{N-k} \xi_{t} \xi_{t+k} .
\end{aligned}
$$


As médias amostrais correspondentes ao segundo e terceiro termo da igualdade acima convergem a zero, quando $N$ tende a infinito, já que as variáveis $\xi_{t}$ e $Y_{t}=V(T(\phi))$ são não-correlacionadas.

As médias amostrais correspondentes ao primeiro termo da igualdade acima já foram analizadas anteriormente com o uso do Teorema Ergódico.

Finalmente, as médias amostrais referentes ao quarto termo convergem a zero, quando $N$ tende ao infinito, já que as variáveis aleatórias $\xi_{t}$ na definição de um ruído branco Gaussiano são não correlacionadas.

Portanto, concluímos que para o processo

$$
Z_{t}=A \cos \left(\omega_{1} t+\phi\right)+\xi_{t},
$$

onde $\frac{\omega_{1}}{2 \pi}$ é irracional e $\left\{\xi_{t}\right\}_{t \in \mathbf{Z}}$ é um ruído branco Gaussiano, as autocovariâncias amostrais de ordem $k$ são estimadores fortemente consistentes para as autocovariâncias de ordem $k$ do processo $\left\{Z_{t}\right\}_{t \in \mathbf{Z}}$.

Ressaltamos que, por simplicidade, o processo $\left\{\xi_{t}\right\}_{t \in \mathbf{Z}}$ foi assumido ser um ruído branco Gaussiano mas as afirmações valem mais geralmente para qualquer ruído ergódico colorido.

Colocamos agora o resultado apresentado acima de forma condensada através do seguinte teorema.

Teorema 6.3: Considere o processo estocástico $\left\{Z_{t}\right\}_{t \in \mathbf{Z}}$ dado pela expressão (4.1) onde o processo $\left\{\xi_{t}\right\}_{t \in \mathbf{Z}}$ é um ruído branco Gaussiano e independente do processo $\left\{Y_{t}^{-}\right\}_{t \in \mathbf{Z}}$. Então, o processo $\left\{Z_{t}\right\}_{t \in \mathbf{Z}}$ é estacionário e ergódico sempre que $\frac{\omega_{1}}{2 \pi}$ é irracional.

A condição de ser irracional é genérica (tem probabilidade um) no conjunto das possíveis freqüências.

O Teorema 6.3 assegura a propriedade de consistência forte dos parâmetros, o que torna lícito tomar médias amostrais dos dados da série temporal para estimar os valores esperados que aparecem na definição da aplicação $\rho(\cdot)$ dada através das expressões (4.2) ou (4.3).

Desta maneira fica demonstrado a justificação do procedimento descrito no Resultado Principal, isto é, dado $\alpha_{0} \in(-1,1), \rho^{k}\left(\alpha_{0}\right)$ estima o coseno de uma certa freqüência, quando $k$ é grande (por exemplo, $k=8$ ). Para obter as outras freqüências podemos proceder em etapas do seguinte modo. Após a localização do co- 
seno da freqüência $\omega_{l}$, aplicamos um filtro passa banda para tirar fora apenas esta freqüência. A série resultante corresponderá a um modelo do tipo (2.1) em que temos agora $p-1$ freqüências. Considerando um outro $\alpha_{0}$ ao acaso, com o procedimento iterativo de atualização de parâmetros acima descrito, agora para a série resultante, estimarrios outro valor $\cos \left(\omega_{i}\right), i \in\{1,2, \ldots, p\}$ e $i \neq l$. Assim, sucessivamente, obtemos todas as freqüências $\omega_{j}$, $j \in\{1,2, \ldots, p\}$.

Não é difícil ver que, quando $p=0$ em (4.1), então $\rho(\alpha)$ é a função identidade. Logo, a iteração $\hat{\rho}^{k}\left(\alpha_{0}\right)$ não move praticamente $\alpha_{0}$. Neste caso, o procedimento chegou ao final e teremos descoberto todas as freqüências e o número $p$.

Para a consistência e a normalidade assintótica dos estimadores $M C$ referimos o leitor aos artigos de $\mathrm{Li}$ e Kedem (1993) e de Li, Kedem e Yakowitz (1991), respectivamente.

\section{Conclusão.}

As freqüências de um modelo de espectro misto podem ser obtidas através de iteração de um ponto $\alpha$ escolhido ao acaso no intervalo das possiveis freqüências. O método apresentado acima é robusto e eficiente. Os vários valores limites $\hat{\rho}^{k}(\alpha)$ são as estimativas dos cosenos das freqüências.

Modelos de freqüência modulada são considerados por Lopes (1994) e Lopes e Kedem (1991) como um caso mais geral da análise feita aqui. Neste caso, existem infinitas freqüências e uma análise matemática mais precisa será objeto de um futuro trabalho.

(Submelido em agoslo de 1993. Revisado em junho de 199/)

\section{Referências}

Cornfeld, I.P., S.V. Fomin \& Ya. G. Sinai 1982. Ergodic Theory. New York: Springer-Verlag.

He, S. \& B. Kedem 1989. "Higher order crossings spectral analysis of an almost periodic random sequence in noise." IEEE Transactions on Information Theory 35:360-370. 
Kedem, B. 1992. "Contraction mappings in mixed spectrum estimation." In Brillinger, D. et al. (eds.), New Directions in Time Series Analysis, Part I. New York: Springer-Verlag, pp. 169-191.

Kedem, B. \& S. Lopes 1992. "Fixed points in mixed spectrum analysis." In Byrnes J. S. et al. (eds.), Probabilistic and Stochastic Methods in Analysis, with Applications (Proceedings of NATO ASI). Massachusetts: Kluwer, pp. 573-591.

Kedem, B. 1994. Time Series Analysis by Higher Order Crossings. New York: IEEE Press.

Kedem, B. \& S. Yakowitz 1991. "On the contraction mapping method for frequency detection." Relatório Técnico 92-45, Systems Research Center, University of Maryland, College Park.

Lamperti, J. 1977. Stochastic Processes - A Survey of the Mathematical Theory. New York: Springer-Verlag.

$\mathrm{Li}, \mathrm{T}-\mathrm{H}$ \& B. Kedem 1993. "Strong consistency of the contraction mapping method for frequency estimation." IEEE Transactions on Information Theory 39: 989-998.

1991. "Asymptotic normality of the contraction mapping estimator for frequency estimation." Relatório Técnico 92-22, Systems Research Center, University of Maryland, College Park.

Lopes, S. 1991. "Spectral analysis in sinusoidal frequency modulated models." Tese de Doutoramento na University of Maryland, Mathematics Department, College Park.

1993. "Amplitude estimation in multiple frequency spectrum." Communications in Statistics, Theory and Methods 22: 2955-2967.

Lopes, S. \& B. Kedem 1994. "Iteration of mappings and fixed points in mixed spectrum analysis." Stochastic Models 10:309-333.

1991. "Sinusoidal frequency modulated spectrum analysis." Relatório Técnico do Instituto de Matemática da UFRGS, Série A, №25, Porto Alegre.

Lopes, S. 1994. "Contraction mapping method in spectral analysis for sinusoidal FM models." A aparecer nas atas do V Congresso Latino Americano em Probabilidade e Estatística Matemática.

Stoer, J. \& R. Bulirsch 1980. Introduction to Numerical Analysis. 
Análise espectral de séries

New York: Springer-Verlag.

Yakowitz, S. 1991. "Some contributions to a frequency location method due to He and Kedem." IEEE Transactions on Information Theory 37:1177-1182. 